Article

\title{
Bidirectional Reflectance Measurement and Reflection Model Fitting of Complex Materials Using an Image-Based Measurement Setup
}

\author{
Aditya Sole *, Ivar Farup $\mathbb{D}^{\mathbb{D}}$, Peter Nussbaum and Shoji Tominaga \\ The Norwegian Colour and Visual Computing Laboratory, Department of Computer Science, \\ Faculty of Information Technology and Electrical Engineering, Norwegian University of Science and \\ Technology, 2815 Gjøvik, Norway; ivar.farup@ntnu.no (I.F.); peter.nussbaum@ntnu.no (P.N.); \\ shoji.tominaga@ntnu.no (S.T.) \\ * Correspondence: aditya.sole@ntnu.no; Tel.: +47-941-655-42
}

Received: 8 October 2018; Accepted: 16 November 2018; Published: 20 November 2018

\begin{abstract}
Materials with a complex visual appearance, like goniochromatic or non-diffuse, are widely used for the packaging industry. Measuring optical properties of such materials requires a bidirectional approach, and therefore, it is difficult and time consuming to characterize such a material. We investigate the suitability of using an image-based measurement setup to measure materials with a complex visual appearance and model them using two well-established reflection models, Cook-Torrance and isotropic Ward. It was learned that the complex materials typically used in the print and packaging industry, similar to the ones used in this paper, can be measured bidirectionally using our measurement setup, but with a noticeable error. Furthermore, the performance of the reflection models used in this paper shows big errors colorimetrically, especially for the goniochromatic material measured.
\end{abstract}

Keywords: BRDF measurement; goniochromatic material; colorimetry

\section{Introduction}

Non-diffuse materials like metallic inks, varnish coatings, and effect paints are widely used in the industry such as for print and packaging to produce a desirable visual appearance of a product. The visual appearance of a material plays an important role in purchase decisions made by the customers.

In traditional printing that uses diffuse material, the color pigments used will absorb part of the incident light, while the rest is diffusely scattered. The perceived color from such pigments is therefore independent of the measurement geometry, and the traditional single geometry $0^{\circ}: 45^{\circ}$ measurements are sufficient to characterize these pigments [1]. Non-diffuse materials like metallic ink used in the print industry contain metal flakes that specularly reflect the light incident on it. Pearlescent pigments (pearl interference pigments) usually consist of thin metal oxide layers on transparent mica platelets. The multi-layered structure of these pearlescent pigments contributes to the variation in visual appearance depending on the illumination and viewing direction. They contain a base layer plus multiple layers of metal oxides with varying refractive indices [2]. The change in visual appearance of these pearlescent pigments with respect to the illumination and viewing direction can be controlled by varying the thickness of the metal oxide layer(s) [3,4]. Colour appearance attributes (like hue, chroma, and brightness) of these pigments are therefore dependent on both illumination and viewing directions. Such materials are called 'goniochromatic' [5,6] and are also used in car paint and the cosmetics industry, apart from the print and packaging industry. 
Because of the directional properties, bi-directional measurements are needed [5] to characterize such materials. ASTM standards $[7,8]$ provide a measurement geometry guide to measure such materials. Instruments performing bidirectional spectral measurements are commercially available and are termed goniospectrophotometers. A goniospectrophotometer usually measures the ratio of the reflected to incident power $\left(\phi_{r} / \phi_{i}\right)$ over a broad range of illumination and viewing directions. The obtained measurement is then used to compute the bidirectional reflectance distribution function (BRDF) [9], $f_{r}$, of a material.

Image-based goniospectrophotometers have been proposed and presented in the past [10-13] to perform bidirectional reflectance measurements in a fast and relatively inexpensive way. However, they are less accurate when compared with slow and expensive techniques similar to the ones used for standardization in metrology. Sole et al. [14] used and evaluated such a measurement setup by measuring fairly diffuse flexible packaging paper samples. The measurements obtained can be used to fit a reflection model (BRDF for an opaque homogeneous material), which will describe the material reflectance properties mathematically for computer graphics rendering or simulating visual appearance attributes.

Many reflection models have been proposed and presented in the past to measure and understand the bidirectional reflectance properties of a given material. Bidirectional reflectance properties of a material are described using a distribution function called the bidirectional reflectance distribution function (BRDF), defined by Nicodemus et al. [9] as:

$$
f_{r}\left(\theta_{i}, \phi_{i} ; \theta_{r}, \phi_{r}, \lambda\right)=\frac{d L_{r}\left(\theta_{i}, \phi_{i}, \theta_{r}, \phi_{r}, E_{i}\right)}{d E_{i}\left(\theta_{i}, \phi_{i}\right)}
$$

where $L_{r}\left(\theta_{i}, \phi_{i}, \theta_{r}, \phi_{r}, \lambda\right)$ is the spectral reflected radiance in the direction $\left(\theta_{r}, \phi_{r}\right), E_{i}\left(\theta_{i}, \phi_{i}, \lambda\right)$ is the spectral irradiance from the direction $\left(\theta_{i}, \phi_{i}\right)$, and $d$ is the differential. Following the ASTM E2175-01 [15] standard and the CIE 175 [16] technical report, the illumination and viewing directions defined above are in relation to the surface normal, called 'anormal' angles.

An overview of different reflection models was provided by Guarnera et al. [17]. Models can be classified into physical-based and phenomenological models. As described in [17], physical models describe the material physically using micro-facets of different sizes and orientations, while phenomenological models are approximations obtained by fitting the measured data using analytical models. Two well-established models, Cook-Torrance (CT) [18] and isotropic Ward (Ward) [19] have been extensively used to study different sample materials.

To fit such reflection models for isotropic materials, it would be ideal to use in-plane measurement data that will cover all the possible combinations of incident $\left(\theta_{i}\right)$ and viewing $\left(\theta_{r}\right)$ directions. A virtual gonioreflectometer simulation software was used in [20] to assess different sets of measurement geometries. It was demonstrated that the measurements made at equispaced-angular grid points led to inefficient sampling and were sub-optimal for the different loss functions used. It was proposed that for accurate sampling, a greater number of measurements should be made in the specular region compared to non-specular areas with the understanding that the diffuse part BRDF of the material varies less with the change in illumination and viewing directions compared to the specular part.

In the situation where we use the measurement setup (used in [14]), a greater number of illumination directions $\left(\theta_{L}\right)$ would result in a higher number of incident and viewing direction combinations, thus giving a dense sampling and possibly a better estimation of the material BRDF. Measuring the samples at a number of $\theta_{L}$ directions, however, adds to the measurement setup complexity. It would therefore be ideal to use a minimum sampling dataset ((that is, a dataset obtained by illuminating the curved samples using a single illumination direction $\left.\left(\theta_{L}\right)\right)$ to estimate the material BRDF successfully. Nielsen et al. [21] presented a novel method to map BRDF space and to optimize for the best sampling direction. They used the MERLdatabase to test the method and concluded that when using an image-based measurement setup, two illumination directions are enough to characterize the BRDF of a given material. Aittala et al. [22] presented a two-shot method to capture spatially-varying BRDF of a textured material using a mobile camera. 
Modeling complex materials present a challenge due to their optical properties, and measuring them bidirectionally using goniospectrophotometers is a time-consuming task. Image-based goniospectrophotometers, similar to the one used by Sole et al. [23,24], can be used for bidirectional measurements, as they can be fast, but might need multiple shots to model such materials due to their goniochromatic and non-diffuse reflectance properties.

In this paper, we investigate the suitability of the image-based measurement setup used in [23-25] to measure such complex materials and model them using well-established reflection models. This setup is well explained in [14,23-25], and we would request the reader to refer to these articles for more details. We use the terminology 'our measurement setup' when referring to this image-based measurement setup. Two reflection models, CT and Ward, are fitted using the measured data, and their performance is compared against a commercially-available goniospectrophotometer (GCMS). Second, we simplify the measurement procedure, when using our measurement setup, by finding the optimal sampling data to fit the reflection models, CT and Ward, for the materials measured in this paper.

\section{Method}

\subsection{Measurement Samples}

Three flexible packaging sample materials, a fairly diffuse chromatic packaging print paper sample generated using an OCE ColorWave 600 plotter, referred to as "Red", one metallic gold thin card board commonly used for decorative purposes in packaging, referred to as "Gold", and a goniochromatic sample (a packaging paper printed using effect pigments and varnish coating), referred to as "Blue-Green", were measured using our measurement setup and the GCMS instrument. Looking at the surface properties, the Red and Blue-Green sample were less homogeneous and appeared rougher compared to the Gold sample, which was smoother and uniform.

These samples showed fairly diffuse (Red) to highly specular reflectance properties (Gold and Blue-Green). The Blue-Green sample was non-diffuse and also goniochromatic in nature. It showed a shift in chromaticity with the change in the viewing direction. Figure 1 shows the spectral shift obtained with the change in illumination and viewing direction for the Blue-Green sample. Figure 2 shows the spectral reflectance of all three samples measured using specular included $\left(d i: 8^{\circ}\right)$ and excluded $\left(d e: 8^{\circ}\right)$ measurement geometry. $d i: 8^{\circ}$ and $d e: 8^{\circ}$ are sphere-based reflectance measurement geometries, as defined in [1], where the sample to be measured is irradiated by an integrating sphere, so that the sample gets irradiated uniformly from all directions within the hemisphere, and the radiation reflected from the sample surface is received at $8^{\circ}$ off the sample normal. The difference between specular included $\left(d i: 8^{\circ}\right)$ and excluded $\left(d e: 8^{\circ}\right)$ measurement is that in specular excluded $\left(d e: 8^{\circ}\right)$ geometry, the radiation reflected in the direction of the receiver from the mirror angle is blocked using a black-trap. No difference was observed in the spectral reflectance curve of the Red sample when comparing the specular included and excluded measurements. However, the same was not the case for the Gold and Blue-Green samples. Along with these samples, a Munsell White N9/sheet (MW) that was produced according to the ANSI standards was measured as a reference white.

\subsection{BRDF Measurement and Reflection Model Fitting}

All three samples were measured using our measurement setup and the GCMS goniospectrophotometer. The measurement output of the GCMS was the radiance factor $\left(\beta_{r}\right)$. It records the spectral radiance factor in the range of $390 \mathrm{~nm}-730 \mathrm{~nm}$ at $10-\mathrm{nm}$ intervals at anormal incident $\left(\theta_{i}\right)$ and reflection $\left(\theta_{r}\right)$ angles in the range of $\left[+80^{\circ},-80^{\circ}\right]$ at $5^{\circ}$ intervals. Please refer to Appendix B in [14] for more details about the GCMS instrument. 


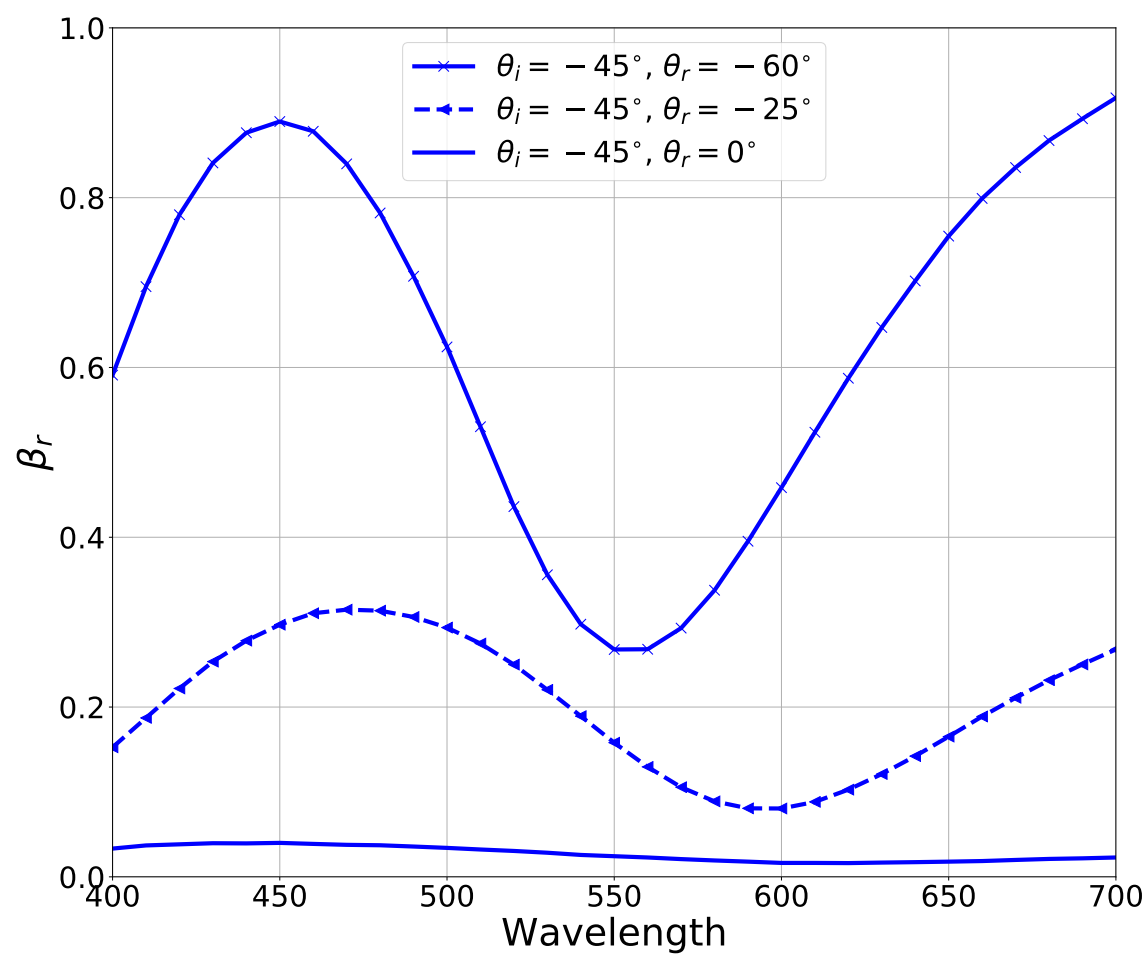

Figure 1. Color shift obtained from the spectral radiance factor measurements of the Blue-Green sample surface using the GCMSinstrument at $\theta_{i}=-45^{\circ}$ and $\theta_{r}=-65^{\circ},-25^{\circ}$ and $0^{\circ}$. Please note: $f_{r}$ can be further calculated using the $f_{r}=\frac{\beta_{r}}{\pi}$ relation [26].

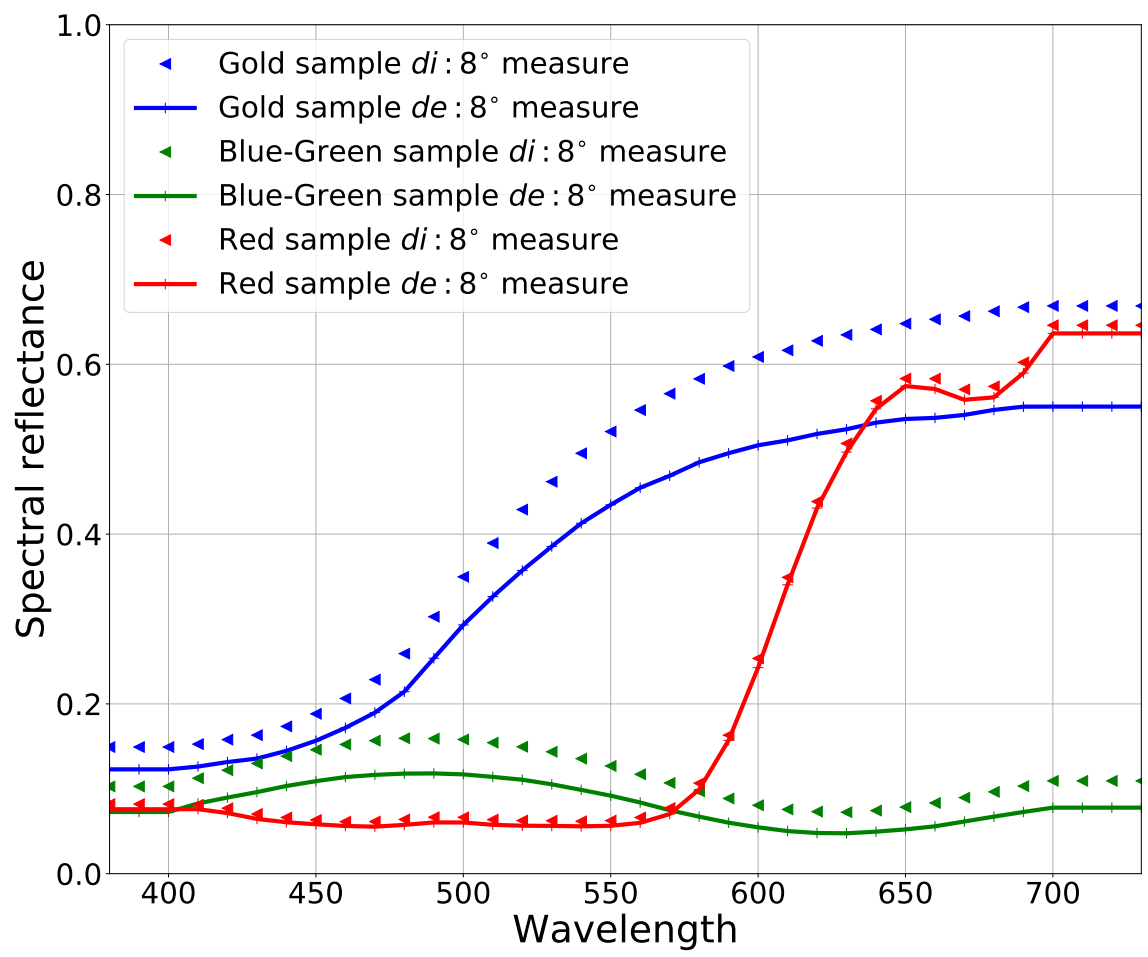

Figure 2. Spectral reflectance measurements using the X-rite SP64 spectrophotometer (with specular included and excluded measurement geometry).

In our measurement setup, the samples were wrapped around a cylinder of known radius and were illuminated and measured respectively using a tungsten point light source and a 
commercially-available digital camera (Nikon D200). The detector and the point light source were at a fixed position from the sample curved onto a cylinder of known radius (for example, light source at $45^{\circ}$ and detector at $\left.0^{\circ}\right)$. Each point, $P$, on the curved sample surface made a corresponding incident $\left(\theta_{i}\right)$ and viewing $\left(\theta_{r}\right)$ angle depending on the illumination direction $\left.\theta_{L}\right)$ of the point light source in the setup. Figure 3 shows the setup in a vector plane. Our measurement setup was well explained in [14,23-25], which can be referred to for more details. As discussed in [27], the captured image records the radiance $\left(L_{r}\left(\theta_{i}, \theta_{r}\right)\right)$ exited from the sample surface in digital values for each camera sensor. It saves a 16-bit raw image without any white balance or gamma correction.

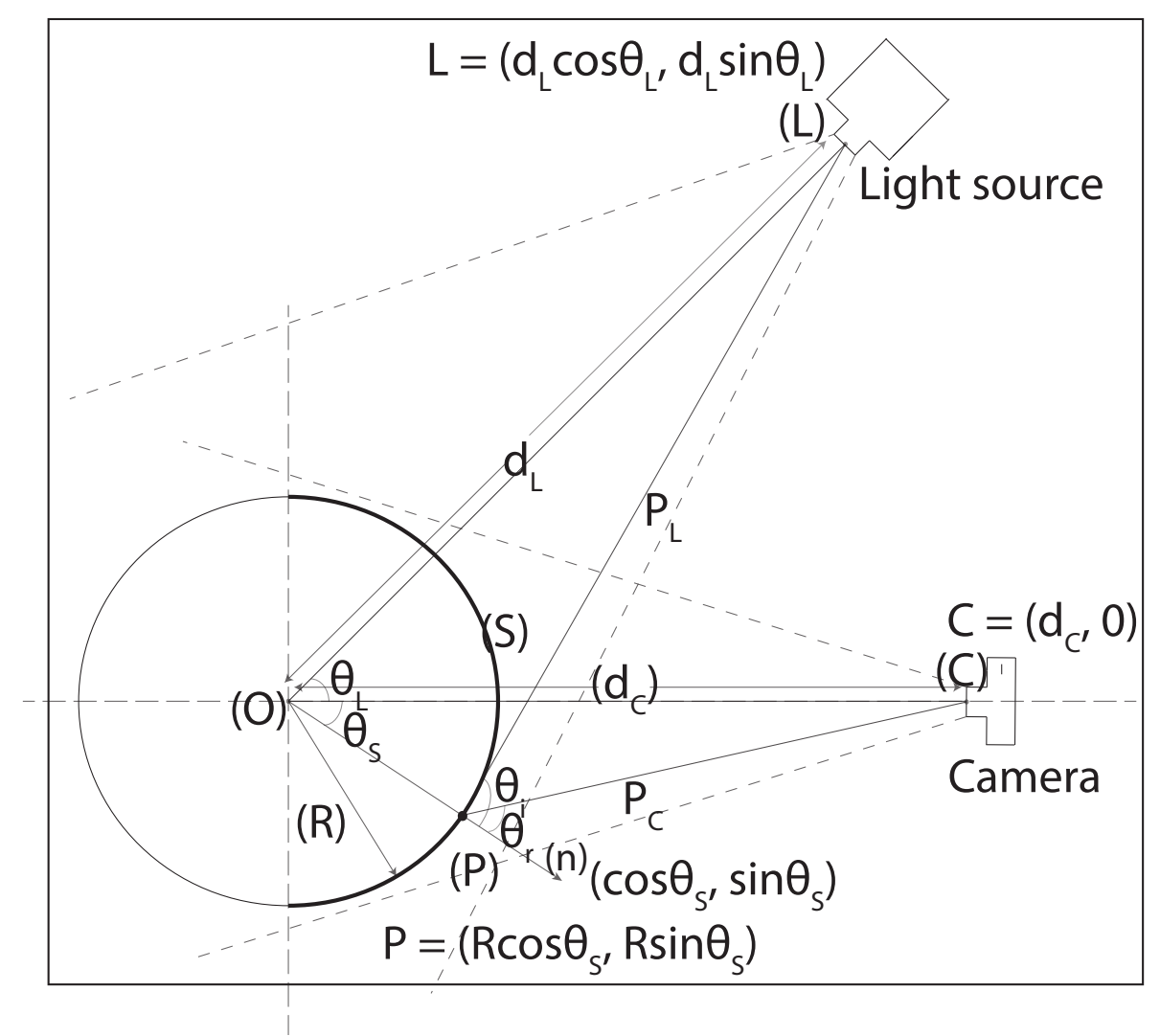

Figure 3. Measurement setup in a vector plane (reprinted with permission of IS\&T: The Society for Imaging Science and Technology, sole copyright owners of CIC22: Twenty-second Color and Imaging Conference) [23].

Each pixel in the captured image corresponds to point $(P)$ on the curved sample surface. Five vertical pixels from the sample center for the given point $(P)$ were averaged. Incident $\left(\theta_{i}\right)$ and viewing $\left(\theta_{r}\right)$ angles at point $(P)$ on the curved sample surface can be calculated using the respective illumination direction $\left(\theta_{L}\right)$ information [23]. Please note that even with a single illumination direction $\left(\theta_{L}\right)$, that is a single position of the point light source, a whole range of incident $\left(\theta_{i}\right)$ and viewing $\left(\theta_{r}\right)$ angles was obtained in a single measurement due to the curvature of the sample and the spatial nature of the imaging sensor. MW and the samples were measured at six illumination directions $\left(\theta_{L}=-20^{\circ}\right.$, $-25^{\circ},-30^{\circ},-35^{\circ},-40^{\circ},-45^{\circ}$ ) (see Figure 4 ). 


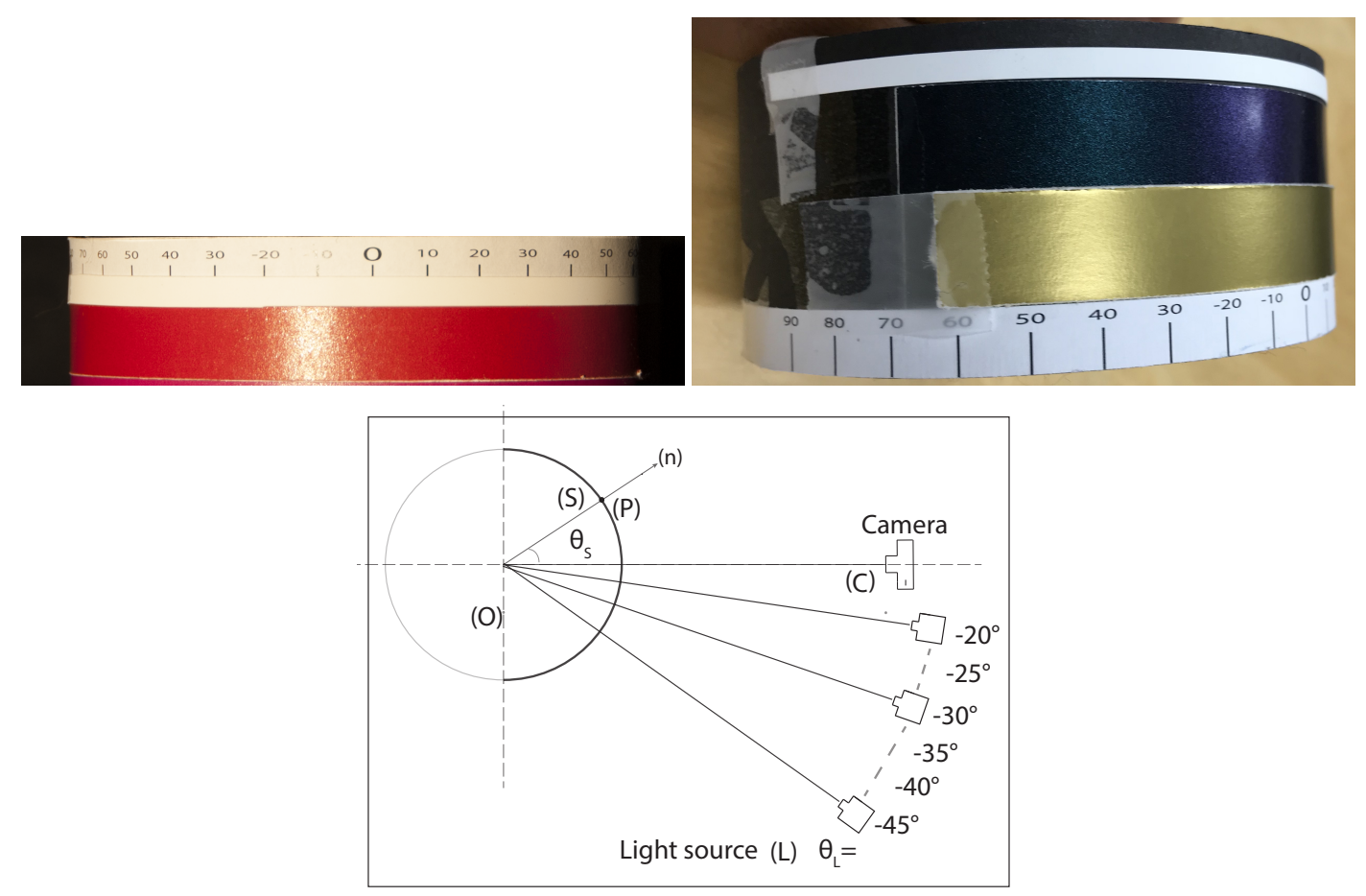

Figure 4. Sample measurement at six different illumination directions $\left(\theta_{L}\right)$ and image captured at $\theta_{L}=-20^{\circ}$.

Being non-diffuse, for the samples Gold and Blue-Green, it was not possible to record the complete dynamic range in a single exposure image. Both samples were therefore captured at multiple exposures by controlling the shutter speed in the range of $1 / 2-1 / 100 \mathrm{~s}$. A high dynamic range image (a radiance map) (HDR) was generated using Debevec and Malik's [28] algorithm. As the obtained HDR image was a radiance map and in order to compare the measurements with the measurements obtained using GCMS, we calculated the radiance factor at the sample surface by using the MW radiance measurements from the same HDR image. As defined in [29] and discussed in [26], the radiance factor $\left(\beta_{r}\right)$ at the material surface is the ratio of the radiance of that material surface in a given direction to that of a perfect reflecting diffuser (PRD) when both are identically irradiated. The generalized definition of $\beta_{r}$ will therefore be as defined in [26] and given in Equation (2).

$$
\beta_{r}\left(\theta_{i}, \phi_{i}, \theta_{r}, \phi_{r}, \lambda\right)=\frac{L_{r}\left(\theta_{i}, \phi_{i}, \theta_{r}, \phi_{r}, \lambda\right)}{L_{r}^{P R D}\left(\theta_{i}, \phi_{i}, \theta_{r}, \phi_{r}, \lambda\right)}
$$

where $L_{r}$ and $L_{r}^{P R D}$ is radiance at the sample and PRD surface, and $\theta$ and $\phi$ are the polar and azimuth angles, respectively. Indexes $i$ and $r$ are incident and reflected radiation. $\lambda$ denotes the wavelength. As defined in [30], PRD is an ideal isotropic diffuser with reflectance equal to unity. It is known that PRD does not exist in the real world, as it is not possible to have a material with such characteristics. In practice, commercially-available reflection standards (such as a Spectralon tile) that have a traceable calibration to a transfer standard at the material supplier are used [26]. As the samples were wrapped around a cylinder and illuminated using a point light source in our measurement setup, using a flat Spectralon tile (similar to how it was used in [25]) will not be precise, as both the wrapped sample and flat Spectralon tile will not be irradiated identically. The Spectralon tile being physically inflexible, the curved sample and the Spectralon tile will be irradiated identically only at a single illumination and viewing direction, thus requiring further assumptions about the homogeneity of the light source used. We therefore use a Munsell White N9/ sheet produced according to ANSI standards to calculate the radiance factor at the sample surface.

As the samples and the MW are wrapped around the cylinder (see Figure 4), one below the other, vertically, any given point $(P)$ on the MW surface will have the same incident $\left(\theta_{i}\right)$ and viewing angle 
$\left(\theta_{r}\right)$ (with respect to the point light source and the detector) as at point $(P)$ on the sample surface. We assume the MW as a PRD, and using the definition of radiance factor $\left(\beta_{r}\right)$ (see Equation (2)), we calculated $\beta_{r}$ at the sample surface using Equation (3).

$$
\beta_{r}\left(\theta_{i} ; \theta_{r}\right)=\frac{L_{r \text { Sample }}\left(\theta_{i}, \theta_{r}\right)}{L_{r M W}\left(\theta_{i}, \theta_{r}\right)}
$$

In Equation (3), $L_{r \text { Sample }}$ and $L_{r M W}$ is the in-plane radiance measurement at the sample surface and the MW surface, respectively. Both surfaces were irradiated identically using the point light source in the measurement setup. The Red sample being a diffuse sample, a single exposure image was sufficient to calculate $\beta_{r}$ using MW measurements and Equation (3).

Figure 5 shows the incident and viewing directions at which the samples were measured using GCMS and our measurement setup. Each diagonal line represents the illumination direction $\left(\theta_{L}\right)$ used in our measurement setup. GCMS measures at $1^{\circ}$ near specular angles, while at $5^{\circ}$ intervals away from the specular direction.

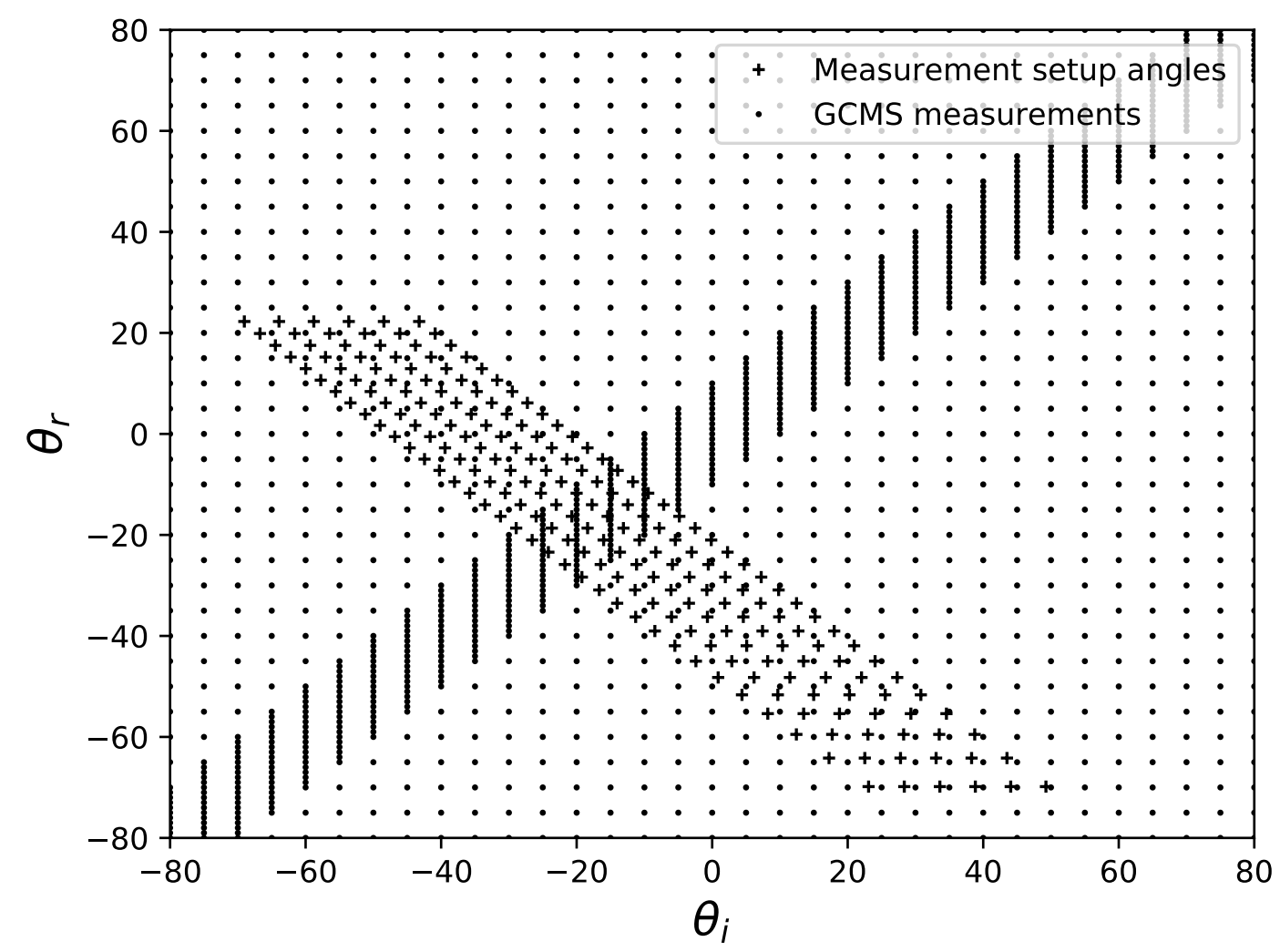

Figure 5. $\theta_{i}$ and $\theta_{r}$ angles at which measurements are performed using our measurement setup and GCMS.

Measurements made using GCMS and our measurement setup had different dimensions. GCMS measurement dimensions were $n \times m \times 35$, where $n$ and $m$ are the incident $\left(\theta_{i}\right)$ and reflection $\left(\theta_{r}\right)$ angles at the sample surface. The measurement unit was a spectral radiance factor in the range of $390 \mathrm{~nm}-730 \mathrm{~nm}$ at 10-nm intervals; whereas, measurements performed using our measurement setup were per pixel, where each pixel corresponds to the curved sample surface point $(P)$, thus making a unique incident and reflection angle relative to the surface normal. The measurement data obtained consisted of 3 channels (R, G, and $\mathrm{B})$. As we used a high resolution camera, the measurement points $(P)$ (or the $\theta_{i}$ and $\theta_{r}$ angle combinations) were many (approximately 1000 pixels horizontally) and different compared to the GCMS measurements. Please note that the measurement setup measurements are sampled at every 20 pixels for visual representation in Figure 5. 
In order to compare both measurements, we converted the radiance factor measurements performed with our measurement setup into a camera color domain (camera RGB domain) using Equation (4).

$$
\begin{array}{r}
\beta_{R_{\text {setup }}}=k \cdot f_{r_{\text {Bsetup }}} \cdot \sum_{\lambda=400 \mathrm{~nm}}^{700 \mathrm{~nm}} \bar{r}(\lambda) \cdot I_{i}(\lambda), \\
\beta_{G_{\text {setup }}}=k \cdot f_{r_{\text {Gsetup }}} \cdot \sum_{\lambda=400 \mathrm{~nm}}^{700 \mathrm{~nm}} \bar{g}(\lambda) \cdot I_{i}(\lambda), \\
\beta_{B_{\text {setup }}}=k \cdot f_{r_{\text {Bsetup }}} \cdot \sum_{\lambda=400 \mathrm{~nm}}^{700 \mathrm{~nm}} \bar{b}(\lambda) \cdot I_{i}(\lambda), \\
k=\frac{100}{\sum_{\lambda 400 \mathrm{~nm}}^{700} \bar{g}(\lambda) \cdot I_{i}(\lambda)}
\end{array}
$$

where $k$ is a normalizing coefficient, $f_{r_{R G B s e t u p}}$ is the sample radiance factor obtained from Equation (3) for each camera channel. $\bar{r}, \bar{g}$, and $\bar{b}$ are the spectral sensitivities of the camera used as a detector, and $I_{i}(\lambda)$ is spectral light intensity normally incident on the sample. Spectral camera sensitivities $(\bar{r}, \bar{g}$, $\bar{b}$ ) were measured using a monochromator as described in [27]. $I_{i}(\lambda)$ was estimated using the relative normalization method described in [31] and implemented in [14].

Similarly, we converted measurements performed using GCMS to the camera RBG domain using Equation (5).

$$
\begin{array}{r}
\beta_{R_{G C M S}}=k \cdot \sum_{\lambda=400 n m}^{700 n m} f_{r_{G C M S}}(\lambda) \cdot \bar{r}(\lambda) \cdot I_{i}(\lambda), \\
\beta_{G_{G C M S}}=k \cdot \sum_{\lambda=400 n m}^{700 n m} f_{r_{G C M S}}(\lambda) \cdot \bar{g}(\lambda) \cdot I_{i}(\lambda), \\
\beta_{B_{G C M S}}=k \cdot \sum_{\lambda=400 n m}^{700 n m} f_{r_{G C M S}}(\lambda) \cdot \bar{b}(\lambda) \cdot I_{i}(\lambda), \\
k=\frac{100}{\sum_{\lambda 400 n m}^{700} \bar{g}(\lambda) \cdot I_{i}(\lambda)}
\end{array}
$$

In Equation (5), $k, \bar{r}, \bar{g}, \bar{b}$, and $I_{i}(\lambda)$ are similar to those used in Equation (4). $f_{r_{G C M S}}$ is the sample radiance factor measured using the GCMS goniospectrophotometer.

To compare sample measurements from both the instruments directly, we can interpolate the camera RGB measurements calculated using GCMS measurements in Equation (5) at the incident and reflection angles of our measurement setup using a standard piece-wise cubic spline interpolation method. The sample measurements obtained can be used to train different reflection models.

Both the $\mathrm{CT}$ and Ward model consist of a diffuse and a specular reflection component along with a roughness parameter that controls the width of the specular component. Considering the diffuse and non-diffuse reflectance properties of the Red and Gold sample, we trained the CT and Ward model by optimizing the diffuse $\left(K_{d}\right)$ and specular reflection coefficient $\left(K_{s}\right)$ individually for each channel $(R, G, B)$, while only a single roughness $(m)$ parameter across all three channels, as shown in Equations (6) and (7) respectively. 


$$
\begin{gathered}
\beta_{p}=\left[\begin{array}{l}
\beta_{p_{R}} \\
\beta_{p_{G}} \\
\beta_{p_{B}}
\end{array}\right]=\left[\begin{array}{l}
K_{s_{R}} \\
K_{s_{G}} \\
K_{s_{B}}
\end{array}\right] \cdot R_{S}+\left[\begin{array}{l}
K_{d_{R}} \\
K_{d_{G}} \\
K_{d_{B}}
\end{array}\right] \\
R_{S}=\frac{F \cdot D \cdot G}{\pi(\mathbf{n} \cdot \mathbf{1})(\mathbf{n} \cdot \mathbf{v})} \\
G=\min \left\{1, \frac{2(\mathbf{n} \cdot \mathbf{h})(\mathbf{n} \cdot \mathbf{v})}{(\mathbf{v} \cdot \mathbf{h})}, \frac{2(\mathbf{n} \cdot \mathbf{h})(\mathbf{n} \cdot \mathbf{1})}{(\mathbf{v} \cdot \mathbf{h})}\right\} \\
\beta_{p}=\frac{1}{m^{2} \cos ^{4} \alpha} e^{-[(\tan \alpha) / m]^{2}} \\
{\left[\begin{array}{l}
\beta_{p_{R}} \\
\beta_{p_{G}} \\
\beta_{p_{B}}
\end{array}\right]=\left[\begin{array}{l}
K_{d_{R}} \\
K_{d_{G}} \\
K_{d_{B}}
\end{array}\right] \cdot \frac{1}{\pi}+\left[\begin{array}{l}
K_{s_{R}} \\
K_{s_{G}} \\
K_{s_{B}}
\end{array}\right] \cdot \frac{1}{\sqrt{\cos \theta_{i} \cos \theta_{r}}} \cdot \frac{e^{\left[-\tan ^{2} \alpha / m^{2}\right]}}{4 \pi m^{2}}}
\end{gathered}
$$

where $R_{s}, D, G$, and $F$ are as defined in Equation (6) and [18]. $F$ is assumed to be one; $\theta_{i}=$ the angle between the illumination direction and normal to the sample surface; $\theta_{r}=$ the angle between the viewing direction and normal to the sample surface; $k_{S_{R, G, B}}=$ the specular reflection coefficient of the sample material per channel; $R_{S}=$ the specular reflectance component, $K_{d}=$ the diffuse reflection coefficient; $\alpha=\mathbf{n} \cdot \mathbf{h}=\cos \left(\left(\theta_{i}-\theta_{r}\right) / 2\right)$ at the given pixel point $(P)$ in the used measurement setup; $\mathbf{n} \cdot \mathbf{l}=\cos \theta_{i} ;$ and $\mathbf{n} \cdot \mathbf{v}=\cos \theta_{r}$.

For the Blue-Green sample, we optimized the roughness coefficient $(m)$ individually for each channel (RGB) along with the specular $\left(K_{s}\right)$ and diffuse $\left(K_{d}\right)$ component, as defined in Equations (8) and (9). $G$ is same as defined in Equations (6).

$$
\begin{gathered}
\beta_{p}=\left[\begin{array}{l}
\beta_{p_{R}} \\
\beta_{p_{G}} \\
\beta_{p_{B}}
\end{array}\right]=\left[\begin{array}{l}
K_{S_{R}} \cdot R_{S_{R}} \\
K_{s_{G}} \cdot R_{S_{G}} \\
K_{S_{B}} \cdot R_{S_{B}}
\end{array}\right]+\left[\begin{array}{l}
K_{d_{R}} \\
K_{d_{G}} \\
K_{d_{B}}
\end{array}\right] \\
R_{S_{R G B}}=\frac{F \cdot D_{R G B} \cdot G}{\pi(\mathbf{n} \cdot \mathbf{1})(\mathbf{n} \cdot \mathbf{v})} \\
\beta_{p}=\left[\begin{array}{l}
\beta_{p_{R}} \\
\beta_{p_{G}} \\
\beta_{p_{B}}
\end{array}\right]=\left[\begin{array}{l}
K_{d_{R}} \\
K_{d_{G}} \\
K_{d_{B}}
\end{array}\right] \cdot \frac{1}{\pi}+\left[\begin{array}{l}
K_{S_{R}} \\
K_{S_{G}} \\
K_{S_{B}}
\end{array}\right] \cdot \frac{1}{\sqrt{\cos \theta_{i} \cos \theta_{r}}} \cdot\left[\begin{array}{l}
\frac{e^{\left[-\tan ^{2} \alpha / m_{R}^{2}\right]}}{4 \pi m_{R}^{2}} \\
\frac{e^{\left[-\tan ^{2} \alpha / m_{G}^{2}\right]}}{4 \pi m_{G}^{2}} \\
\left.\frac{e^{\left[-\tan ^{2} \alpha / m_{B}^{2}\right]}}{4 \pi m_{B}^{2}}\right]
\end{array}\right]
\end{gathered}
$$

The Nelder-Mead down-hill simplex algorithm [32] was used to optimize the coefficients using the RMS error $\left(\beta_{E r r}\right)$ as defined in Equation (10). 


$$
\beta_{E r r}=\sqrt{\left(\beta_{R_{\text {Pmea }}}-\beta_{R_{\text {Pest }}}\right)^{2}+\left(\beta_{G_{\text {Pmea }}}-\beta_{G_{\text {Pest }}}\right)^{2}+\left(\beta_{B_{\text {Pmea }}}-\beta_{B_{\text {Pest }}}\right)^{2}}
$$

$\beta_{p_{R_{\text {mea }}}}, \beta_{p_{\text {Gmea }}}$ and $\beta_{p_{B_{\text {mea }}}}$ are the radiance factor measurements in the camera RGB domain obtained using Equations (4) and (5) for the three channels, while $\beta_{{R_{\text {est }}}}, \beta_{p_{G_{e s t}}}, \beta_{p_{B_{e s t}}}$ are the radiance factor measurements (again in the camera RGB domain) of the sample calculated using the fitted CT and Ward models.

\subsection{Optimal Sampling Dataset}

When collecting the sampling dataset using our measurement setup, it was important to use the best and least possible illumination directions $\left(\theta_{L}\right)$ to fit the reflection models successfully. This would help towards reducing the measurement complexity and performing fast measurements. We, therefore, fit both the models using the sampling dataset collected from all combinations (63 in total) of the six illumination directions $\left(\theta_{L}\right)$, respectively. The obtained model parameters were then used to estimate the bidirectional reflectance measurements in the camera RGB domain $\left(\beta_{R G B}\right)$.

\section{Results}

Figure 6 shows the measured (using GCMS) and predicted (using the fitted reflection models) camera RGB G-channel data.
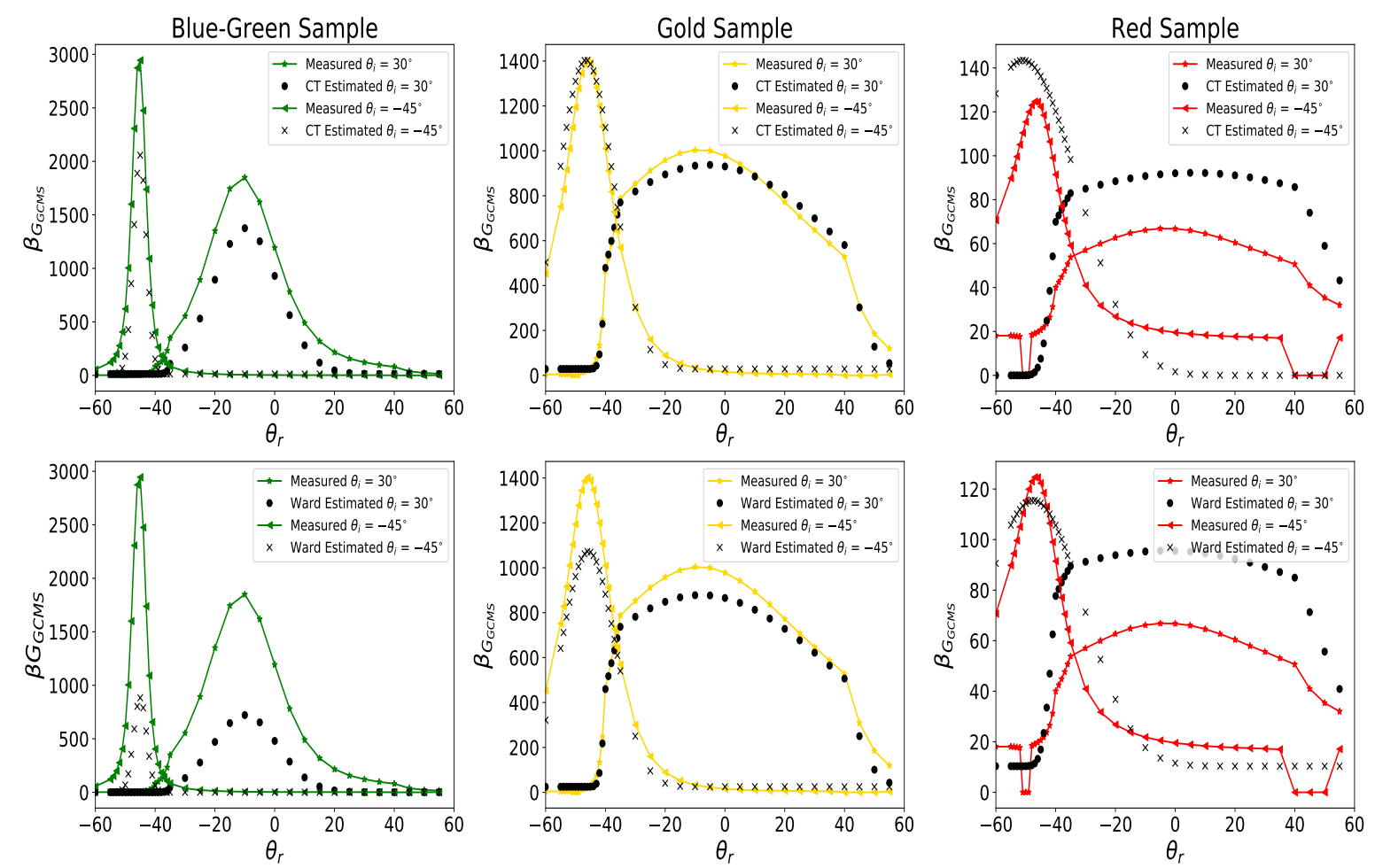

Figure 6. G-channel camera RGB data measured using GCMS and estimated using models fitted with the data set captured with $\theta_{L}=-25^{\circ}$ using our measurement setup.

The relative error $\left(\Delta E r r_{P}\right)$ was calculated, using Equation (11), between camera RGB data that were calculated using the measured data and predicted using the fitted reflection models for the respective channel.

$$
\Delta \operatorname{Err}_{P}=\frac{\sum_{p}\left|\beta_{P_{\text {mea }}}-\beta_{P_{e s t}}\right|}{\sum_{p} \beta_{P_{\text {mea }}}}
$$


In Equation (11), $\beta_{P_{\text {mea }}}$ is camera RGB data calculated from measurements, and $\beta_{P_{\text {est }}}$ is camera RGB data estimated using the fitted reflection models.

Using measurements made with our measurement setup, we fit both the reflection models, CT and Ward, with sampling data obtained from all the possible combinations (63 in total) of the incident light directions $\left(\theta_{L}=-20^{\circ}--45^{\circ}\right)$, respectively.

Figure 7 shows box-and-whisker plots for average relative error $\left(\Delta E r r_{P}\right)$ calculated using Equation (11) for the G-channel between camera RGB data calculated from GCMS measurements and data estimated using both the reflection models fitted with different combinations of sampling data. The box-and-whiskers plots show that the error variation was reduced to some extent when a bigger sampling dataset was used (from a single illumination direction $\left(\theta_{L}\right)$ to two $\theta_{L}$ directions) to fit the models. However, in many cases, the optimization algorithm converged to a local minimum instead of a global minimum when finding the reflection model parameters using the cost function given in Equation (10). This can also be seen in the results shown in Figure 7.
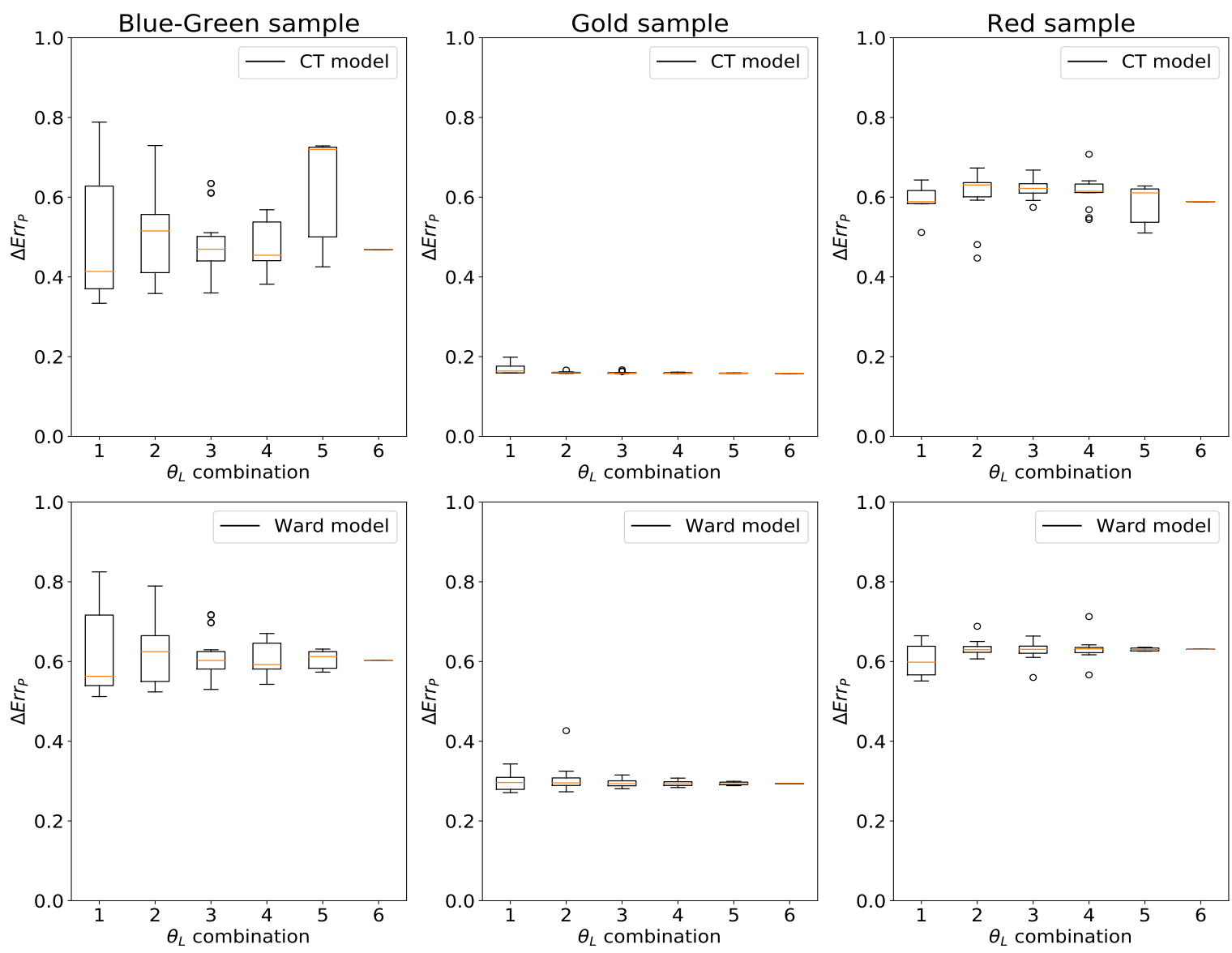

Figure 7. Box-and-whisker plots showing $\Delta E r r_{P}$ between G-channel camera RGB data calculated from GCMS measurements and data estimated using both models of all the samples. The orange line in the boxes shows the median error value obtained between the $\theta_{L}$ combinations used for model fitting. Circle dots are large $\Delta E r r_{P}$ error obtained (approximately three-times the range of error variation (boxes) obtained). CT, Cook-Torrance.

One of the objectives of our measurement setup being bidirectional reflectance measurement of goniochromatic materials, we colorimetrically evaluated the setup by calculating CIE1976 $u^{\prime} v^{\prime}$ uniform chromaticity coordinates [1] from the obtained camera RGB data. We converted the obtained camera RGB data to the CIEXYZ colorimetric space using a transformation matrix $(\hat{M}) . \hat{M}$ was calculated using the camera spectral sensitivities and CIE $2^{\circ}$ color matching functions, as described 
in [27]. The obtained CIEXYZ values were then transformed into CIE1976 $u^{\prime} v^{\prime}$ uniform chromaticity coordinates using Equation (12).

$$
u^{\prime}=\frac{4 \cdot X}{X+15 \cdot Y+3 \cdot Z^{\prime}}, \quad v^{\prime}=\frac{9 \cdot Y}{X+15 \cdot Y+3 \cdot Z}
$$

Figure 8 shows the CIE1976 $u^{\prime} v^{\prime}$ values from measured (using GCMS) and predicted (using the fitted reflection models) values for all the samples. The total number of measurements being many, we present below CIE1976 $u^{\prime} v^{\prime}$ plots for all the samples measured (with GCMS) and predicted using reflections models fitted with the dataset captured with our measurement setup at a single illumination direction $\left(\theta_{L}=-45^{\circ}\right)$.
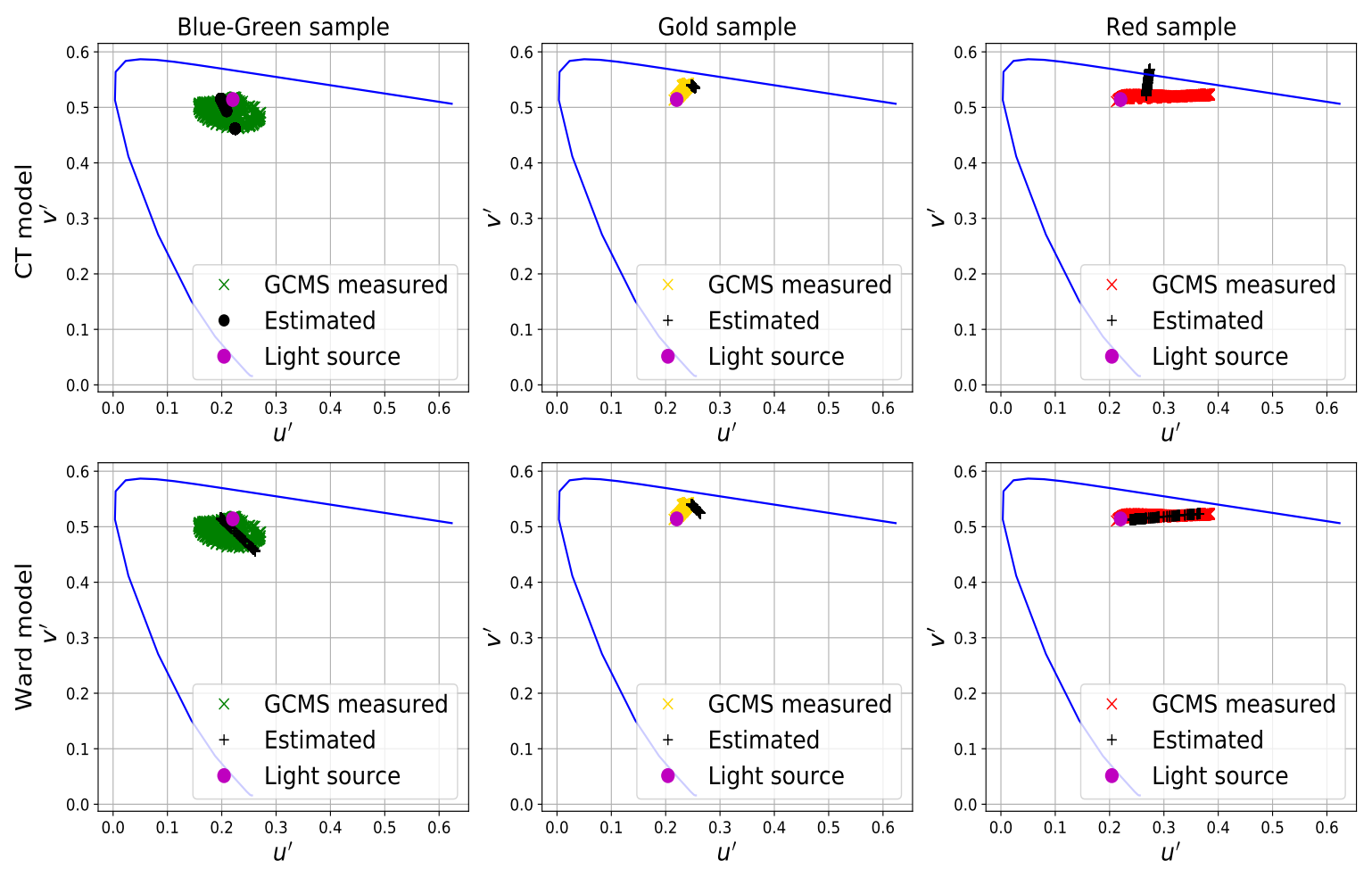

Figure 8. CIE1976 $u^{\prime} v^{\prime}$ uniform chromaticity coordinates calculated using Equation (12) for all the samples measured with GCMS and estimated from reflection models trained using the dataset obtained from our measurement setup with one illumination direction $\left(\theta_{L}=-45^{\circ}\right)$.

The relative error $\left(\Delta E_{u^{\prime} v^{\prime}}\right)$ was calculated using Equation (13) between CIE1976 $u^{\prime} v^{\prime}$ coordinates obtained from the measured and predicted camera RGB data.

$$
\Delta E_{u^{\prime} v^{\prime}}=\left(\frac{\sqrt{\left(u_{\text {mea }}^{\prime}-u_{\text {est }}^{\prime}\right)^{2}}}{u_{\text {mea }}^{\prime}}+\frac{\sqrt{\left(v_{\text {mea }}^{\prime}-v_{\text {est }}^{\prime}\right)^{2}}}{v_{\text {mea }}^{\prime}}\right)
$$

where $u_{m e a}^{\prime}$ and $v_{\text {mea }}^{\prime}$ are the CIE1976 $u^{\prime} v^{\prime}$ coordinates obtained from the measured $\beta_{r}$ data and $u_{e s t}^{\prime}$ and $v_{\text {est }}^{\prime}$ were obtained from $\beta_{r}$ estimated using the reflection models. Figure 9 shows box-and-whisker plots for the average relative error calculated using Equation (13) for all the samples. Samples were measured using GCMS and predicted using both reflection models fitted with the datasets obtained using all (63) combinations of $\theta_{L}$ directions in our measurement setup. 

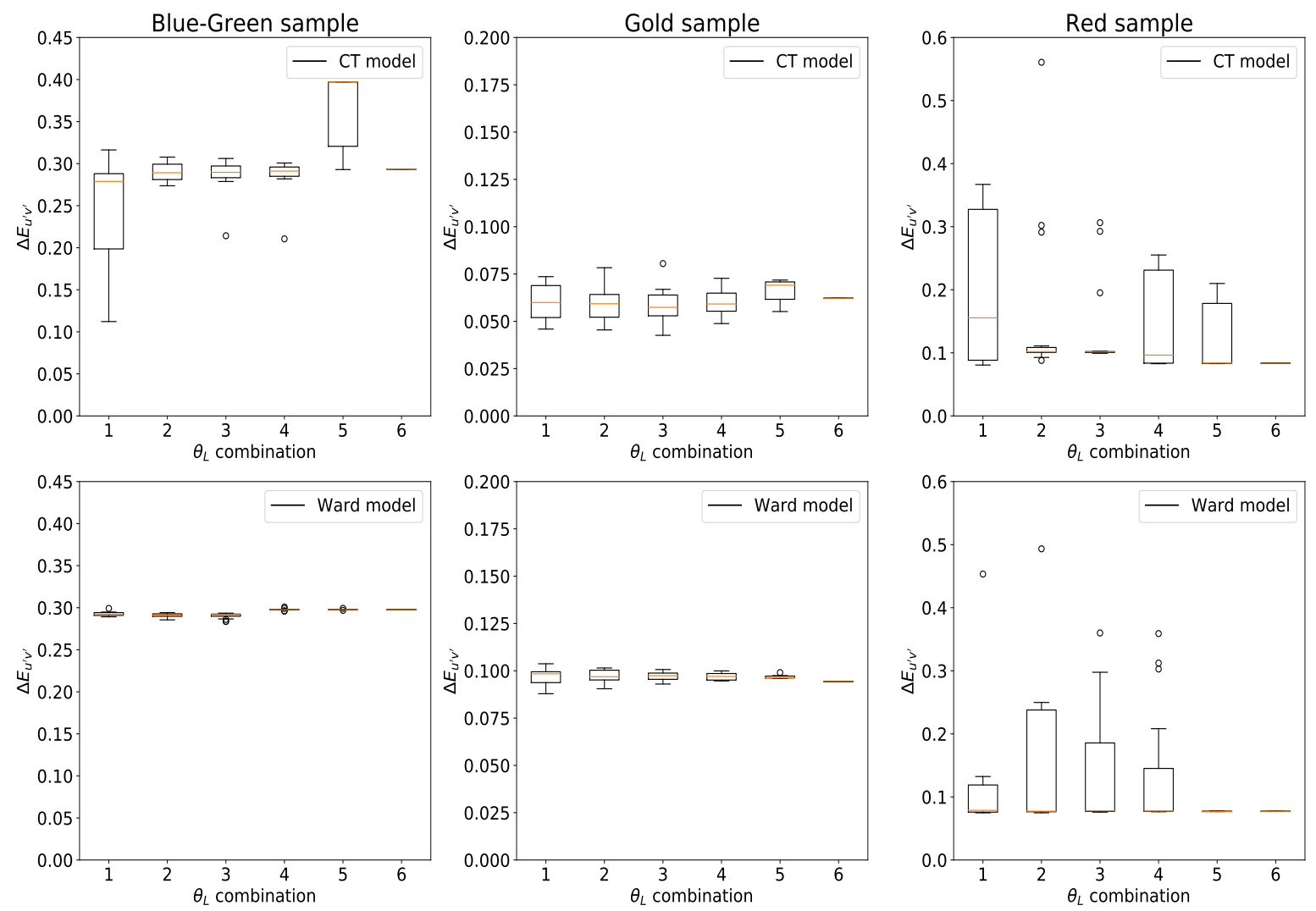

Figure 9. Box-and-whisker plots showing average $\Delta E_{u^{\prime} v^{\prime}}$ between camera RGB data calculated from GCMS measurements and data estimated using both models of all the samples. The orange line in the boxes shows the median error value obtained between the $\theta_{L}$ combinations used for model fitting. Circle dots are large $\Delta E_{u^{\prime} v^{\prime}}$ error obtained (approximately three-times the range of error variation (boxes) obtained).

The median error remained fairly constant for all three samples with the increase in the number of measurements to fit the reflection models. Adding more illumination directions did not improve the fitting performance colorimetrically. Modeling goniochromatic materials using the CT and Ward model was difficult given the nature of these models, and it would be more appropriate to use a mixture of BTFand BRDF models.

\section{Discussion}

This paper investigates the suitability of measuring materials with a complex visual appearance bidirectionally using our measurement setup. The setup is evaluated against measurements made using a commercially available goniospectrophotometer (GCMS). Two well-established reflection models are fitted using sampling data obtained from our measurement setup. Figure 6 shows the G-channel camera RGB data measured and estimated using the GCMS instrument and the fitted reflection models, respectively. It can be seen that the performance of the CT model was better compared to the Ward especially for the Blue-Green material and Gold sample. Estimated BRDF values match well with the measurements for the Gold sample for non-grazing illumination and viewing directions.

Box-and-whisker plots shown in Figure 7 show a similar result, with the performance of CT being better for all the samples. The variation in the error value was much higher (maximum error value $\left(\Delta E r r_{p}\right)$ of 0.8$)$, for the Blue-Green sample G-channel measurements when we used a minimum sampling dataset (that is, the dataset collected using a single $\theta_{L}$ in our measurement setup). The median relative error obtained was around 0.4 (using the CT model), which is a fairly big error. 
The error was even higher for the Ward model (median relative error of almost 0.6). Similar results were obtained for the Red sample with a median relative error around 0.6 for both, CT and Ward, reflection models. For the Gold sample, the median relative error was approximately 0.2 for both the reflection models, which is much smaller compared to the Blue-Green and Red sample. Using a bigger sampling dataset for any of the samples or the reflection models did not reduce the error variation significantly. In many cases, the optimization algorithm did converge to a local minimum instead of a global minimum, thus adding to the uncertainty in fitting the models.

Overall, the relative error was large for Blue-Green and Red material compared to Gold. Some of the possible reasons for having less error for the Gold sample are the material being smoother and uniform. Furthermore, the Gold sample can be called uni-modular, which shows a big change in intensity compared to hue and chroma for different illumination $\left(\theta_{i}\right)$ and viewing $\left(\theta_{r}\right)$ directions.

The CIE $1976 u^{\prime} v^{\prime}$ chromaticity coordinates were calculated from the camera RGB data for colorimetric evaluation. The performance of both models was similar for the Blue-Green and Gold samples, but with less variation in the relative $\Delta E_{u^{\prime} v^{\prime}}$ error when using the Ward model. Colorimetrically, the performance was not good for either of the models for the Blue-Green sample. Variation in the relative error $\left(\Delta E_{u^{\prime} v^{\prime}}\right)$ was small with the Ward model compared to CT for the Gold and Blue-Green samples. Colorimetrically, both models struggled to predict the Blue-Green sample. One of the possible reasons for this is that we had constant diffuse reflection components $\left(K_{d_{R G B}}\right)$ trained using the measured data, while the Blue-Green sample showed a shift in color depending on the viewing direction. Another observation made using the analysis was that the sample measurements showed retro-reflection, which cannot be modeled using CT and Ward. Furthermore, the relative error $\left(\Delta E r r_{p}\right)$ obtained was maximum at the grazing angles, which the models struggled to predict.

The samples also being non-diffuse (similar to the Gold sample) added the further complexities of a dynamic range and the need to capture a high dynamic range image. Capturing an HDR image adds to measurement error and uncertainty, as the accuracy of the radiance map will depend on the fusion algorithm. The need to capture an HDR image also reduces the measurement speed and limits the use of such a measurement setup to measure the BRDF of complex materials.

A Munsell White sheet (MW) was used as a reference white standard when calculating the radiance factor from the measurements obtained using our measurement setup. MW is a white paper material reproduced by following the ANSI specifications according to the Munsell color system. In practice, commercially-available reflection standards such as a Spectralon tile are used in reference instruments or commercially-available goniospectrophotometers like GCMS. The tiles are spectrally calibrated with high accuracy, and double beam measurements are performed to record the bidirectional radiance factor on the sample surface, resulting in a very precise measurement. The MW sheet is not as precise as a Spectralon tile as a reference white standard and can contribute to measurement error. As discussed in detail in [26], performing absolute calibration would be the most appropriate method to obtain accurate radiance factor measurements. This, however, is difficult to implement in the industry, where performing measurements in a fast and inexpensive way is expected. When performing measurements using our measurement setup, using a Spectralon tile that can be curved onto the cylinder along with the material to be measured would be one of the methods to perform BRDF calculations. This way, it would be possible to follow the definition to have both the sample and the Spectralon tile irradiated identically. It was not possible to obtain a Spectralon tile that could be used in this way, and therefore, we attempted in this paper to use a Munsell white N9/sheet that was produced according to the ANSI standards. Furthermore, if we use a flat Spectralon tile, it would only be valid for a single incident $\left(\theta_{i}\right)$ and viewing $\left(\theta_{r}\right)$ angle combination in our measurement setup, and we would have to make further assumptions about the homogeneity of the light source. With the MW, this was avoided. Using MW as a reference white standard in our measurement setup is a known source of error, which needs to be addressed in future work.

There are many factors that contribute to the total error when fitting both models. Some of the error sources with respect to our measurement setup were identified in [14]. HDR image capture 
will further add to the setup error when measuring complex materials along with the reference white standard to be used for radiance factor calculations. This will need further study, measurements and analysis.

\section{Conclusions}

Complex materials with different reflectance properties are measured using our measurement setup. The setup is evaluated using two well-established reflections models. The measurement procedure, when using our measurement setup, is simplified by evaluating optimal sampling data that can be used to fit the reflection models for the complex materials measured. A commercial goniospectrophotometer is used to evaluate the performance of the model fitting and to investigate the feasibility to use our measurement setup to measure complex materials.

Though the error obtained is fairly big, the estimated BRDF of the non-diffuse and goniochromatic material measured look similar to the GCMS measurements, especially at the non-grazing angles. Colorimetrically, both models struggle with the Blue-Green sample. The Gold sample showed less relative error compared to the Red and Blue-Green samples, possibly due to its surface properties being smoother and homogeneous compared to the Red and Blue-Green samples. Using more sampling data (in terms of illumination directions $\left(\theta_{L}\right)$ ) did not reduce the median error significantly, though the error variation was reduced to some extent. We learned that the complex materials typically used in the print and packaging industry, similar to the ones used in this paper, can be measured using our measurement setup, but with a noticeable error.

Author Contributions: Supervision, I.F. and P.N.; Writing—original draft, A.S.; Writing—review \& editing, A.S., I.F., P.N. and S.T.

Funding: This work was supported by the MUVAppProject N-250293, funded by the Research Council of Norway.

Acknowledgments: We would like to thank and acknowledge support of Reiner Eschbach, Jean-Baptiste Thomas, and Giuseppe Claudio Guarnera, at the Norwegian Colour and Visual Computing Laboratory, in discussions and suggestions regarding the structure of this paper.

Conflicts of Interest: The authors declare no conflict of interest.

\section{References}

1. Colorimetry; CIE15.2; CIE Standard; CIE Publication: Paris, France, 2004.

2. Maile, F.J.; Pfaff, G.; Reynders, P. Effect pigments-Past, present and future. Prog. Org. Coat. 2005, 54, 150-163. [CrossRef]

3. Klein, G.A. Industrial Color Physics; Springer Series in Optical Sciences; Springer: New York, NY, USA, 2010.

4. Tomić, I.; Dedijer, S.; Novaković, D.; Jurič, I. Artificial neural networks for optimizing camera-based color measurements of prints enhanced with pearlescent pigments. Color. Technol. 2018, 134, 364-372. [CrossRef]

5. McCamy, C. Observation and measurement of the appearance of metallic materials. Part I. Macro appearance. Color Res. Appl. 1996, 21, 292-304. [CrossRef]

6. Eugène, C. MEASUREMENT OF "total visual appearance": A CIE challenge of soft metrology. In Proceedings of the 12th IMEKO TC1 and TC7 Joint Symposium on Man, Science and Measurement, Annecy, France, 3-5 September 2008; pp. 61-65.

7. Standard Practise for Multiangle Color Measurement of Interference Pigments; ASTM-E2539; ASTM Standard; American Society for the Testing of Materials: West Conshohocken, PA, USA, 2012.

8. Standard Practise for Multiangle Color Measurement of Metal Flake Pigmented Materials; ASTM-E2194; ASTM Standard; American Society for the Testing of Materials: West Conshohocken, PA, USA, 2012.

9. Nicodemus, F.E.; Richmond, J.; Hsia, J.J.; Ginsberg, I.W.; Limperis, T. Geometrical Considerations and Nomenclature for Reflectance; National Bureau of Standards; US Department of Commerce: Washington, DC, USA, 1977.

10. Rong Lu, J.; Koenderink, J.; Kappers, A.M.L. Optical properties (bidirectional reflection distribution functions) of velvet. Appl. Opt. 1998, 37, 5974-5984. 
11. Marschner, S.R.; Westin, S.H.; Lafortune, E.P.F.; Torrance, K.E.; Greenberg, D.P. Image-Based BRDF Measurement Including Human Skin. In Proceedings of the Eurographics Workshop, Granada, Spain, 21-23 June 1999; pp. 139-152.

12. Tominaga, S.; Tanaka, N. Estimating reflection parameters from a single color image. IEEE Comput. Gr. Appl. 2000, 20, 58-66. [CrossRef]

13. Matusik, W.; Pfister, H.; Brand, M.; McMillan, L. A Data-Driven Reflectance Model. ACM Trans. Gr. 2003, 22, 759-769. [CrossRef]

14. Sole, A.; Farup, I.; Nussbaum, P.; Tominaga, S. Evaluating an image-based bidirectional reflectance distribution function measurement setup. Appl. Opt. 2018, 57, 1918-1928. [CrossRef] [PubMed]

15. Standard Practice for Specifying the Geometry of Multiangle Spectrophotometers; ASTM-E2175; ASTM Standard; American Society for the Testing of Materials: West Conshohocken, PA, USA, 2013.

16. A Framework for the Measurement of Visual Appearance; CIE175; Technical Report; International Commission on Illumination: Vienna, Austria, 2006.

17. Guarnera, D.; Guarnera, G.; Ghosh, A.; Denk, C.; Glencross, M. BRDF Representation and Acquisition. Comput. Gr. Forum 2016, 35, 625-650. [CrossRef]

18. Cook, R.L.; Torrance, K.E. A Reflectance Model for Computer Graphics. ACM Trans. Gr. 1982, 1, 7-24. [CrossRef]

19. Ward, G.J. Measuring and Modeling Anisotropic Reflection. SIGGRAPH Comput. Gr. 1992, 26, $265-272$. [CrossRef]

20. Langovoy, M.; Schmähling, F.; Wübbeler, G. Numerical comparison of sampling strategies for BRDF data manifolds. Measurement 2016, 94, 578-584. [CrossRef]

21. Nielsen, J.B.; Jensen, H.W.; Ramamoorthi, R. On Optimal, Minimal BRDF Sampling for Reflectance Acquisition. ACM Trans. Gr. 2015, 34. [CrossRef]

22. Aittala, M.; Weyrich, T.; Lehtinen, J. Two-shot SVBRDF capture for stationary materials. ACM Trans. Gr. 2015, 34, 110:1-110:13. [CrossRef]

23. Sole, A.; Farup, I.; Tominaga, S. An image based multi-angle method for estimating reflection geometries of flexible objects. In Color and Imaging Conference; Society for Imaging Science and Technology: Springfield, VA, USA, 2014; Volume 2014, pp. 91-96.

24. Sole, A.S.; Farup, I.; Tominaga, S. An image-based multi-directional reflectance measurement setup for flexible objects. In Measuring, Modeling and Reproducing Material Appearance 2015; Segovia, M.V.O., Urban, P., Imai, F.H., Eds.; International Society for Optics and Photonics: Bellingham, WA, USA, 2015; Volume 9398, p. 93980J. [CrossRef]

25. Sole, A.; Farup, I.; Nussbaum, P. Evaluating an image based multi-angle measurement setup using different reflection models. Electron. Imaging 2017, 2017, 101-107. [CrossRef]

26. Höpe, A.; Hauer, K.O. Three-dimensional appearance characterization of diffuse standard reflection materials. Metrologia 2010, 47, 295. [CrossRef]

27. Sole, A.; Farup, I.; Tominaga, S. Image based reflectance measurement based on camera spectral sensitivities. Electron. Imaging 2016, 2016, 1-8. [CrossRef]

28. Debevec, P.E.; Malik, J. Recovering High Dynamic Range Radiance Maps from Photographs. In Proceedings of the 24th Annual Conference on Computer Graphics and Interactive Techniques, Los Angeles, CA, USA, 11-15 August 2008; ACM Press/Addison-Wesley Publishing Co.: New York, NY, USA, 1997; pp. 369-378. [CrossRef]

29. Palmer, J.; Grant, B.G. The Art of Radiometry; SPIE Press Bellingham: Washington, DC, USA, 2010; ISBN 978-0-8194-7245-8.

30. CIE. International Lightning Vocabulary 4th edn CIE Publication 17.4; CIE Publication: Paris, France, 1987.

31. Li, H.; Foo, S.C.; Torrance, K.E.; Westin, S.H. Automated three-axis gonioreflectometer for computer graphics applications. Opt. Eng. 2006, 45, 043605. [CrossRef]

32. Nelder, J.A.; Mead, R. A Simplex Method for Function Minimization. Comput. J. 1965, 7, 308-313. [CrossRef]

(C) 2018 by the authors. Licensee MDPI, Basel, Switzerland. This article is an open access article distributed under the terms and conditions of the Creative Commons Attribution (CC BY) license (http://creativecommons.org/licenses/by/4.0/). 